\title{
Estruturação Genética das Populações de Duas Espécies de PeiXes RECIFAIS do ATOl das Rocas E da Costa do CEARÁ.
}

\author{
JoÃo EDUARDO P. FREITAS ${ }^{1}$, \\ MARIA ElisabeTH DE ARAúJoº, \\ ANTÔNIO MATEO SOLÉ-CAVA ${ }^{3}$
}

\begin{abstract}
RESUMO
O Atol das Rocas, que recentemente foi declarado pela UNESCO patrimônio natural da humanidade, é o único do Atlântico Sul e dista 144 milhas náuticas (266,68 Km) da costa brasileira. A corrente predominante nesta localidade é a Sul Equatorial que se estende até a costa do Ceará e posteriormente da origem a corrente das Guianas. As condições oceanografias e estratégias reprodutivas das várias famílias de peixes associados aos recifes de Rocas e da Costa do Ceará podem influenciar na dispersão larval e, conseqüentemente, caracterizar a estruturação genética dessas populações. Foram analisadas geneticamente populações do Atol das Rocas e Costa do Ceará, de duas espécies de peixes recifais com desova pelágia e longo período de duração larval. São elas: Acanthurus chirurgus e Cephalopholis fulva. A metodologia utiliza foi eletroforese de isoenzimas. Para A. chirurgus os resultados indicaram a presença de estoques pesqueiros diferenciados entre as duas populações. O mesmo não foi encontrado para as populações de $C$. fulva que apresentaram fluxo gênico irrestrito.
\end{abstract}

Palavras-chave: Acanthurus chirurgus, Cephalopholis fulva, disperção larval, fluxo gênico, isoenzimas, Atol das Rocas.

\section{Abstract \\ Genetic Structure of Two Reef Fishes Species From Atol das Rocas and Ceará Coast Populations.}

The Atol das Rocas, which recently was declared a Humanity Natural Heritage by UNESCO, is the unique of South Atlantic, and distance 144 nautical miles $(266,68 \mathrm{Km})$ from Brazilian coast. The predominant current in this locality is the South Equatorial's Current that arrive at Ceará coast and, subsequently origins the Guiana’s Current. The oceanographic conditions and reproduction strategies of many fishes' families associated to Atol das Rocas and Ceará coast' reefs may influence on larval dispersion and, consequently, characterize the genetic structure of those populations. Tissues' samples of two species from those populations were analyzed genetically. Those species, Acanthurus chirurgus e Cephalopholis fulva, have pelagic spawns and a long period of larval duration. The method used was isozymes' electrophorese. Results show that it has two different fisheries stocks of A. chirurgus in those two populations, while C. fulva's populations present a continuous genetic flow between the Atol and the coast.

Key words: Acanthurus chirurgus, Cephalopholis fulva, gene flow, isozyme,dispersal, Atol das Rocas

\footnotetext{
${ }^{1}$ Eng. de Pesca e Pesquisador do Grupo de Ictiologia Marinha Tropical (IMAT), Universidade Federal do Ceará, Departamento de Eng. de Pesca BL: 827 Av. Humberto Monte S/N Campos do Pici: C.P 12.168 - CEP 60356-000 Fortaleza-CE e Instituto de Ciências do Mar (LABOMAR). E-mail: eduardojpf@hotmail.com
}

Tropical Oceanography, Recife: v. 31, n. 2, p. 171-180, 2003 
${ }^{2}$ Professora adjunta do Departamento de Oceanografia (CTG) da Universidade Federal de Pernambuco - Av. da Arquitetura, s/n, Cidade Universitária - CEP 50.740-550, Recife -PE. Coordenadora do Grupo de Ictiologia Marinha Tropical (IMAT) da Universidade Federal do Ceará,

${ }^{3}$ Professor adjunto do Departamento de Genética da Universidade Federal do Rio de Janeiro. Laboratório de Biodiversidade Molecular, Departamento de Genética, Instituto de Biologia, Bloco A CCS - Ilha do Fundão. CEP 21941-490 - Rio de Janeiro - RJ.

\section{INTRODUÇÃo}

Praticamente metade das espécies de vertebrados é constituída por peixes, que ocupam a maioria dos hábitats aquáticos, possuem grande mobilidade e muitas estratégias de reprodução (Planes, 1998). Os ambientes recifais abrigam as comunidades de peixes mais diversas (LoweMcConnel, 1999). Como os peixes recifais costumam viver intimamente associados aos recifes, de maneira relativamente sedentária, sua dispersão é feita principalmente no estágio planctônico (Sale, 1980). Hourigan \& Reese (1987) destacam duas principais estratégias reprodutivas para peixes recifais: A primeira apresenta ovos demersais, com proteção de adultos, baixa fecundidade (de 25 a 10.000 ovos) e um curto período larval (15 a 30 dias). A segunda estratégia resulta em uma fase planctônica, com alta fecundidade (100 a 1.000 .000 ovos) e longo período larval (20 a 80 dias). É esperado que, devido à sua menor capacidade de dispersão, espécies que usam a primeira estratégia tenham populações geneticamente estruturadas, enquanto que espécies do segundo grupo tenham populações mais homogêneas. Por exemplo, estima-se que $85 \%$ da variação na estrutura genética de populações de peixes da grande barreira de coral da Austrália pode ser explicada pelo tipo de dispersão larval (Planes et al., 1998).

Neste estudo estima-se o grau de estruturação gênica, de populações de duas espécies de peixes entre a ilha oceânica do Atol das Rocas (que fica a $267 \mathrm{~km}$ da costa) e a região costeira brasileira, no litoral do Ceará. As espécies estudadas foram Acanthurus chirurgus (Bloch, 1787) e Cephalopholis fulva (Linnaeus, 1758), pertencentes às famílias Acanthuridae e Serranidae, respectivamente. Estas espécies são potencialmente boas dispersoras, por apresentarem desova pelágica em grande quantidade, estão entre as mais abundantes no Atol das Rocas (Rosa \& Moura, 1995) e na ictiofauna recifal do nordeste brasileiro (Araújo et al., 2000; Floeter et al., 2001).

Os marcadores moleculares escolhidos para este estudo foram as alozimas, que têm sido usadas com sucesso no estudo da diferenciação genética entre populações de invertebrados (Thorpe \& Solé-Cava, 1994; Thorpe et al., 2000) e peixes (Doherty et al., 1995; Carvalho \& Pitcher, 1995;

Ward, 2000). Apesar de mais de 250 espécies de peixes marinhos já terem sido estudadas geneticamente por alozimas, poucas delas foram de espécies de recifes de coral (Planes, 1998).

\section{Espécies estudadas}

Acanthurus chirurgus, conhecida vulgarmente por Lanceta ou Cirurgião, tem sua distribuição definida para os dois lados do Atlântico (Hoese \& Moore, 1998; Floeter \& Gasparini, 2000), ocupando as partes mais rasas de recifes de corais, e bancos de fanerógamas marinhas (Nagelkerken et al., 2000). Não foram encontrados dados sobre a estratégia reprodutiva desta espécie, mas a espécie próxima $A$. triostegus (Linnaeus, 1758) apresenta uma fecundidade estimada de 100.000 ovos e um tempo de duração do período larval de 60 dias (Planes, 1998).

Cephalopholis fulva, conhecida vulgarmente por Piraúna, tem a sua distribuição compreendida entre o Caribe, sul da Florida, Golfo do México e sul do Brasil (Humann, 1999; Floeter et al., 2001). Esta espécie caracteriza-se por apresentar fecundação externa, com ovos pelágicos e uma fecundidade estimada entre 150.000 e 282.000 ovos (Heemstra \& Randall, 1993).

Tropical Oceanography, Recife: v. 31, n. 2, p. 171-180, 2003 


\section{Áreas estudadas}

Trabalhos recentes mostram que, das 320 espécies de peixes recifais encontradas em águas brasileiros, aproximadamente 18\% estão restritas ao sudoeste do Atlântico (Floeter \& Gasparini, 2001). O endemismo de algumas espécies pode ser devido ao isolamento das ilhas oceânicas em relação ao continente (Lacoste \& Salamon, 1973; Floeter \& Gasparini, 2000; Floeter \& Gasparini, 2001). O Atol das Rocas e Fernando de Noronha são as ilhas oceânicas brasileiras que possuem a maior semelhança com a margem continental (53\% da ictiofauna compartilhada), mas também apresentam um endemismo considerável (6.9\%) na sua fauna ictiológica (Floeter \& Gasparini 2000). Para o Atol das Rocas, que recentemente foi declarado patrimônio natural da humanidade (UNESCO), estão descritas 147 espécies, incluindo a Stegastes rocasensis (Emery, 1972) um pomacentrídeo endêmico do conjunto insular oceânico Rocas-Fernando de Noronha (Rosa \& Moura, 1997).

As formações recifais brasileiras são encontradas desde a costa do Maranhão até a costa do Rio de Janeiro, sendo que a maioria destas está assentada sobre substrato rochoso não coralino (Maida \& Ferreira, 1997). É o caso do litoral cearense, onde os arrecifes encontrados são formados pela cimentação do óxido de ferro e carbonato de cálcio, resultando em substrato de arenito (Smith \& Morais, 1984). Na costa cearense estão distribuídos cerca de 50 pontos de ambientes recifais segundo as cartas náuticas 600 e 700. A ictiofauna associada aos ambientes recifais cearenses foi caracterizada por Araújo et al. (2000), que catalogaram 77 espécies das quais 28 eram novas ocorrências para o estado.

Nas proximidades de Fernando de Noronha e Atol das Rocas, a corrente Sul Equatorial subdivide-se em dois ramos: O ramo norte, que origina a corrente das Guianas e o ramo sul, que dá origem à corrente do Brasil. A morfologia oceânica revela um alinhamento de vulcões submarinos no sentido Leste-Oeste (figura 1), constituído pelos Morro do Caruru (CE), bancos oceânicos (com profundidades de até 50 metros em pontos mais rasos), Atol das Rocas e Arquipélago de Fernando de Noronha (Morais, 1969).

Este trabalho analisa populações de duas espécies de peixes recifais Acanthurus chirurgus e Cephalopholis fulva para avaliar a homogeneidade entre as populações do Atol das Rocas e a costa do Ceará.

\section{MATERIAL E MÉtodos}

A licença para pesquisa na Reserva Biológica do Atol das Rocas foi concedida pelo Instituto Brasileiro do Meio Ambiente e dos Recursos Naturais Renováveis (IBAMA), segundo o processo $\mathrm{n}^{\circ}$ 02001,007850/99-68. O número de indivíduos coletados em Rocas limitou-se a cinco exemplares de cada espécie por se tratar de uma Reserva Biológica, mas, para a costa do Ceará foram coletados vinte indivíduos de cada espécie. As artes de pesca utilizadas foram armas de caça submarina e puçás, objetivando minimizar danos ao substrato e para garantir a seletividade.

Foram coletados tecidos de músculo estriado dos exemplares selecionados. As amostras foram colocadas em criotubos e conservadas em nitrogênio líquido até o momento das análises de laboratório. Após a retirada das amostras de tecido, todos os exemplares foram fixados em formol e transportados para o Laboratório onde foram identificados segundo as chaves de Cervigón (1966), Figueiredo \& Menezes (1980); Humann (1999) e Figueiredo \& Menezes (1985). Os espécimes estão organizados na Coleção Ictiológica da Universidade Federal do Ceará.

A metodologia para o estudo de isoenzimas aplicada neste trabalho foi a eletroforese horizontal em gel de amido a 12,5\% desenvolvida por Smithies (1959), com posterior coloração histoquímica específica para cada sistema isoenzimático. As eletroforeses transcorreram em tampão

Tropical Oceanography, Recife: v. 31, n. 2, p. 171-180, 2003 
Tris-Citrato $\mathrm{pH} 8,0$ a uma voltagem de $80 \mathrm{~V}$ e $45 \mathrm{~mA}$, com o tempo de duração variando de 6 a 8 horas (Solé-Cava \& Thorpe, 1986). As enzimas testadas estão apresentadas na legenda da tabela I e os tampões utilizados nas revelações foram Tris-HCl pH 8.0 e o Tris-maleato pH 5.3 (Manchenko, 1994)

As freqüências genotípicas foram obtidas a partir da interpretação dos padrões das bandas e, a partir destas, foram estimadas as freqüências gênicas (Tabela I). Foram também calculados os níveis de variabilidade gênica (heterozigosidade) e as identidades e distâncias gênicas não viciadas (Nei, 1978). Uma outra medida de diferenciação interpopulacional usada foi o índice de endocruzamento, $\mathrm{F}_{\mathrm{ST}}$ (Wright, 1978). Para o cálculo dos parâmetros acima descritos, utilizou-se o programa BIOSYS (Swofford \& Selander, 1981). A significância de $\mathrm{F}_{\mathrm{ST}}$, contra a hipótese nula da panmixia $\left(\mathrm{H}_{0}: \mathrm{F}_{\mathrm{ST}}=0\right)$ foi verificada com o teste do $\chi^{2}$ de Waples (1987).

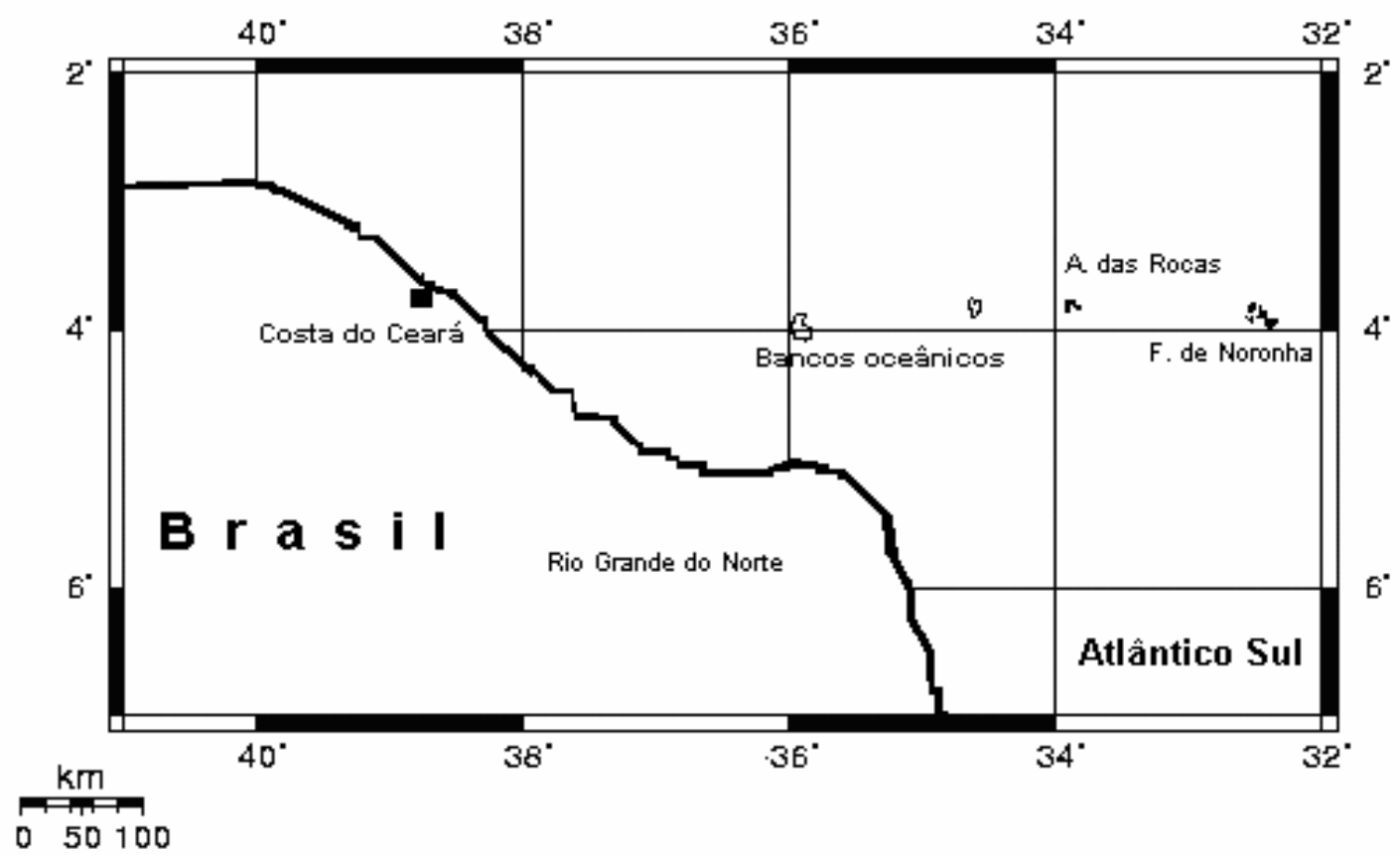

Figura 1 - Área de trabalho destacando o Atol da Rocas, Costa do Ceará e o alinhamento de bancos oceânicos e do Arquipélago de Fernando de Noronha em direção a continente.

\section{RESULTADOS}

As freqüências gênicas para todos os loci estudados estão apresentadas na tabela I. Nenhum loco apresentou desvios significativos nas suas proporções genotípicas em relação ao esperado para populações em equilíbrio de Hardy-Weinberg (teste exato de Fisher, $\mathrm{P}<0,05$ depois de uma correção seqüencial de Bonferroni; Lessios, 1992). Os níveis de heterozigosidade $\left(\mathrm{H}_{\mathrm{e}}\right)$, foram maiores em $A$. chirurgus $\left(\mathrm{H}_{\mathrm{e}}=0,128\right.$ no Atol das Rocas, e $\mathrm{H}_{\mathrm{e}}=0,220$ no Ceará) do que em $C$. fulva $\left(\mathrm{H}_{\mathrm{e}}=0,025\right.$ no Atol das Rocas e $\mathrm{H}_{\mathrm{e}}=0,036$ no Ceará).

As populações de Cephalopholis fulva se apresentaram muito semelhantes quanto às suas freqüências gênicas (Identidade gênica, $\mathrm{I}=1,000 ; \mathrm{F}_{\mathrm{ST}}=0,048 \mathrm{P}>0,05$ ). As populações de Acanthurus chirurgus, apresentaram uma identidade gênica alta $(\mathrm{I}=0,987)$, típica de populações 
co-específicas (Thorpe \& Solé-Cava, 1994) mas apresentaram-se estruturadas genéticamente $\left(\mathrm{F}_{\mathrm{ST}}=\right.$ 0,063; $\mathrm{P}<0,05 ; \mathrm{H}_{\mathrm{o}}$ da panmixia pode ser rejeitada).

Tabela I - Freqüência gênica para todos os loci estudados nas duas localidades.

\begin{tabular}{|c|c|c|c|c|c|}
\hline Lo & $\cos$ & ACANTHURU & RGUS & СЕРНА & IS FULVA \\
\hline & & CEARÁ & A. RocAs & CEARÁ & A. RocAs \\
\hline Adh & & & & & \\
\hline & $\mathrm{N}$ & 18 & 5 & & \\
\hline & A & 0,083 & 0,100 & ND & ND \\
\hline & B & 0,639 & 0,639 & & \\
\hline & C & 0,278 & 0,200 & & \\
\hline Est-1 & & & & & \\
\hline & $\mathrm{N}$ & 13 & 5 & & \\
\hline & A & 0,038 & 0,000 & ND & ND \\
\hline & B & 0,962 & 1,000 & & \\
\hline Gdh & & & & & \\
\hline $\mathbf{N}$ & & 19 & 5 & & \\
\hline & A & 1,000 & 1,000 & ND & ND \\
\hline Ian & $\mathrm{N}$ & 19 & 5 & 19 & 5 \\
\hline & A & 0,132 & 0,000 & 0,947 & 1,000 \\
\hline & B & 0,105 & 0,000 & 0,053 & 0,000 \\
\hline & C & 0,763 & 1,000 & & \\
\hline Ldh-1 & & & & & \\
\hline & $\mathrm{N}$ & 19 & 5 & 19 & 5 \\
\hline & A & 0,053 & 0,000 & 1,000 & 1,000 \\
\hline & B & 0,947 & 1,000 & & \\
\hline Ldh-2 & & & & & \\
\hline & $\mathrm{N}$ & 10 & 5 & & \\
\hline & A & 0,050 & 0,000 & ND & ND \\
\hline & B & 0,950 & 1,000 & & \\
\hline & & Ceará & A. Rocas & Ceará & A. Rocas \\
\hline Mdh-1 & & & & & \\
\hline & $\mathrm{N}$ & 18 & 5 & & \\
\hline & A & 0,806 & 0,900 & ND & ND \\
\hline & B & 0,194 & 0,100 & & \\
\hline Mdh-2 & & & & & \\
\hline & $\mathrm{N}$ & 17 & 5 & & \\
\hline & A & 0,324 & 0,100 & ND & ND \\
\hline & B & 0,676 & 0,900 & & \\
\hline $\mathrm{Me}$ & & & & & \\
\hline & $\mathrm{N}$ & 19 & 5 & 19 & 5 \\
\hline & A & 0,447 & 0,100 & 1,000 & 0,875 \\
\hline & B & 0,553 & 0,900 & 0,000 & 0,125 \\
\hline Mpi & & & & & \\
\hline & $\mathrm{N}$ & 19 & 5 & 19 & 5 \\
\hline & A & 1,000 & 1,000 & 1,000 & 1,000 \\
\hline Pgd & & & & & \\
\hline & $\mathrm{N}$ & 19 & 5 & 14 & 5 \\
\hline & A & 1,000 & 1,000 & 0,036 & 0,000 \\
\hline & B & & & 0,964 & 1,000 \\
\hline Pgm & & & & & \\
\hline & $\mathrm{N}$ & 18 & 4 & 19 & 5 \\
\hline & A & 0,917 & 0,750 & 1,000 & 1,000 \\
\hline & B & 0,028 & 0,000 & & \\
\hline & $\mathrm{C}$ & 0,056 & 0,250 & & \\
\hline Sod & & & & & \\
\hline & $\mathrm{N}$ & & & 19 & 5 \\
\hline & A & ND & ND & 1,000 & 1,000 \\
\hline
\end{tabular}


Álcool desidrogenase - 1.1.1.1 (Adh); $\alpha$-esterase - 3.1.1.1 ( $\alpha$-Est); Glutamato desidrogenase 1.4.1.2 (Gdh); Isocitrato desidrogenase - 1.1.1.42 (Idh); Lactato desidrogenase - 1.1.1.27 (Ldh); Malato desidrogenase - 1.1.1.37 (Mdh); Enzima málica - 1.1.1.40 (Me); Manose fosfato isomerase - 5.3.1.8 (Mpi), Fosfoglicodesidrogenase - 1.1.4.4 (Pgd), Fosfoglicose isomerase - 5.3.1.9 (Pgi); Fosfoglicomutase - 2.7.5.1 (Pgm); Superoxido dimutase - 1.15.1.1 (Sod). N = Tamanho da amostra e ND = ausência de resultados satisfatórios.

\section{DISCUSSÃO}

Neste trabalho observou-se que, enquanto parece existir um fluxo gênico irrestrito (panmixia) entre as populações do Atol das Rocas e do Ceará do serranídeo Cephalopholis fulva, o mesmo não acontece com as populações de Acanthurus chirurgus, que apresentaram restrições ao fluxo gênico entre as duas regiões.

Embora nenhum artigo sobre dados isoenzimáticos de Acanthurus chirurgus tenha sido encontrado, os trabalhos de Planes (1993a); Planes (1993b) e Planes et al., (1998) avaliaram populações da espécie $A$. triostegus da Austrália e da Polinésia francesa, relatando resultados satisfatórios para 17 loci examinados. Esses autores encontraram níveis de heterozigosidade $(\mathrm{h}=$ $0,116)$ e diferenciação gênica $\left(\mathrm{F}_{\mathrm{ST}}=0,049 ; \mathrm{P}<0,05\right)$ entre populações dessa espécie de nível bastante semelhante às encontradas aqui com $A$. chirurgus $\left(\mathrm{h}=0,174 ; \mathrm{F}_{\mathrm{ST}}=0,063 ; \mathrm{P}<0,05\right)$.

A conclusão que se pode chegar no presente trabalho, ainda que com uma amostragem bastante limitada, é semelhante à obtida nos trabalhos com A. triostegus, ou seja, de que as duas regiões parecem possuir estoques pesqueiros diferenciados. No entanto, estudos recentes com DNA mitocondrial (citocromo b) foram feitos com A. chirurgus do Atlântico, e não encontraram diferenças significativas entre amostras do Brasil, da Ilha de Assunção e do Panamá (Rocha et al., 2002). Claramente novas populações dessa espécie devem ser estudadas, através de marcadores nucleares e mitocondriais, antes que possam ser tomadas conclusões sobre o estado de suas populações.

Nenhuma publicação que trate especificamente de Cephalopholis fulva foi encontrada, mas Planes et al., (1998) examinaram 13 loci de Epinephelus merra (Bloch, 1793), espécie também pertencente à família Serranidae. Esses autores encontraram, uma baixa heterozigosidade $(\mathrm{h}=$ $0,065)$ e uma homogeneidade populacional $\left(\mathrm{F}_{\mathrm{ST}}=0,000 ; \mathrm{P}>0,05\right)$ entre as amostras analisadas, valores semelhantes aos obtidos para $C$. fulva $\left(\mathrm{h}=0,030 ; \mathrm{F}_{\mathrm{ST}}=0,048 ; \mathrm{P}>0,05\right)$. Essa alta homogeneidade poderia ser um artefato, devido à baixa variabilidade desses serranídeos, já que a diferenciação populacional, antes de ser atingido o equilíbrio migração/deriva, depende basicamente da variância nas freqüências gênicas (Ward \& Skibinski, 1985; Whitlock \& McCauley, 1999). No entanto, um outro estudo com Epinephelus striatus, usando microssatélites, que são marcadores muito mais polimórficos do que alozimas (Carvalho \& Hauser, 1998), também encontrou uma alta homogeneidade populacional ao longo de todo o Caribe (Stevenson et al., 1998).

É possível, portanto, que a alta homogeneidade observada entre as populações de Cephalopholis fulva esteja, de fato, associada a uma capacidade de dispersão e produção larval maior nessas espécies (Planes et al., 1998; Ward, 2000). Um outro fenômeno que pode estar auxiliando a homogeneidade entre as populações desta espécie de Rocas e da costa do Ceará pode ser a ação das correntes, no caso específico a corrente Sul-Equatorial. De acordo com Doherty et al. (1994) fenômenos oceânicos deste tipo podem atuar como carreadores de larvas entre diversas regiões. Outro ponto relevante é fato de Cephalopholis fulva estar distribuída no Caribe e ao longo de toda a província brasileira (Floeter \& Gasparini, 2000), evidenciando uma grande capacidade de dispersão.

\section{AgRAdeCimentos:}

Ao IBAMA, através da Representação Estadual do Rio Grande do Norte e chefia da Reserva Biológica do Atol das Rocas, pela infra-estrutura utilizadas nas atividades no Atol.

Tropical Oceanography, Recife: v. 31, n. 2, p. 171-180, 2003 
A todos os companheiros de Campo (durante as 5 temporadas em Rocas), em especial a Daniel Alexandrino Pimenta.

\section{REFERÊNCIA BIBLIOGRAFICA}

ARAÚJO, M.E., CUNHA F.E.A.; CARVALHO R.A.A.; FREITAS, J.E.P.; NOTTINGHAM, M.C.; BARROS B.M.N. Ictiofauna marinha do estado do Ceará, Brasil: II. Elasmobranchii e Actinopiterygii de arrecifes de arenito da região entre marés. Arquivos de ciencias do mar.

Fortaleza, v. 33, p.133-138, 2000.

CARVALHO, G. R.; HAUSER. L. Advances in the molecular analysis of fish population structure. Italian Journal of Zoology. v.65, p.21-33, 1998.

CARVALHO, G. R.; PITCHER, T. J. Molecular genetics in fisheries. Chapman \& Hall, London,1995.

CERVIGÓN, F. M. Los peces marinos de Venezuela. Fondo de Cultura Cientifica, Caracas,1966.

DOHERTY, P. J.; MATHER, P.; PLANES, S. Acanthochromis polyacanthus, a fish lackiing larval dispersal, has genetically differentiated populations at local and regional scales on the Great Barrier Reef. Marine Biology, v.121, p.11-21,1994.

DOHERTY, P. J.; PLANES, S.; MATHER, P. Gene flow and larval duration in 7 species of fish from the Great-Barrier-Reef. Ecology, New York, v. 76, n. 8, p. 2373-2391, dez.1995.

FIGUEIREDO, J. L.; MENEZES N. A. Manual de Peixes Marinhos do Sudeste do Brasil. III. Teleostei (2). São Paulo: EDUSP, 1980. 90 p.

FLOETER, S. R.; GASPARINI. J. L. The southwestern Atlantic reef fish fauna: composition \& zoogeographic patterns. Journal of Fish Biology. v. 56 p.1099-1114, 2000.

FLOETER, S. R.; GASPARINI, J. L.; ROCHA, L. A.; FERREIRA, C. E. L.; RANGEL, C. A.; FEITOZA, B. M.; NUNAN, G. W. Brazilian reef fish fauna: checklist and remarks. BioBase Project. 2001. Disponível em: www.biobase.org.

FLOETER, S. R.; Gasparini, J. L. Brazilian endemic reef fishes. Coral Reefs. V.19, p. 292, 2001.

HEEMSTRA, P. C.; RANDALL, J. E. Groupers of the World. (Family Serranidae,Subfamily Epinephelidae). FAO Fish. Sinopse, n. 125, v.16, 1993.

HOESE, H. D.; R. H. MOORE. Fishes of the Gulf of Mexico, Texas, Louisiana, and adjacent waters. A\&M University Press, Texas: 422p 1998.

HOURIGAN, T. F.; REESE E. S. Mid-ocean isolation and the evolutoin fo Hawaiian reef fishes. Trends in Ecology and Evolution. v. 2, p. 187-191, 1987.

HUMANN, P. Reef Fish Identification, 2. ed. Florida: New World Publications, 1999. 396 p.

LACOSTE, A.; SALAMON, R. Biogeografia. Barcelona: Oikos-Tau, 1973. 271 p.

Tropical Oceanography, Recife: v. 31, n. 2, p. 171-180, 2003 
LESSIOS, H. A. Testing Electrophoretic Data For Agreement With Hardy-Weinberg Expectations. Marine Biology. v. 112, p. 517-523, 1992.

LEWE-MCCONNEL, R. H. Estudos ecológicos de comunidades de peixes tropicais. Tradução Vazzoler A. E. A., Agostinho, A. A., Cunnhingham P. T. M. São Paulo: EDUSP, 1999. 382p: Título original: Ecological studies in tropical fish communities.

MAIDA, M.; B. P. FERREIRA. Coral Reefs of Brazil: am Overview. In: International Coral Reef Symposium, 8th., 1997 Proceedings International Coral Reef Symposium, v. 1, p 263-274.

MANCHENKO, G. P. Handbook of detection of enzymes on electrophoretic gels. CRC Press Inc., Ann Arbor, Michigan.1994.

MORAIS, J. O. Aspectos correlativos de geologia litoral e submarina do Nordeste do Brasil. Arquivos de Ciências do Mar, Fortaleza, v. 9, n. 2, p. 127-131, dez. 1969

NAGELKERKEN, I.; VAN DER VELDE, G.; GORISSEN, M. W.; MEIJER, G. J.; VAN'T HOF, T.; DEN HARTOG, C. Importance of mangroves, seagrass beds and the shallow coral reef as a nursery for important coral reef fishes, using a visual census technique. Estuarine Coastal \& Shelf Science v. 51, p. 31-44, 2000.

NEI, M. Estimation of average heterozigosity and genetic distance from a small number of individuals. Genetics, v. 89, p. 583-590, 1978.

PLANES, S. Evidence of differentiated stocks of acanthurus-triostegus (pisces, Acanthuridae) in French-Polynesia, using electrophoretic data. Fisheries Research. Amsterdã, v.16, n. 4, p. 287-299, mai. 1993a.

PLANES, S. Genetic differentiation in relation to restricted larval dispersal of the convict surgeonfish Acanthurus-triostegus in French-Polynesia. Marine Ecology-Progress Series. v. 98, n. 3, p. 237-246 aug.1993b.

PLANES, S. Genetic diversity and dispersal capabilities in marine fish. Evolutionary Biology, New York , v. 30, p. 252-298.1998.

PLANES, S.; PARRONI, M.; CHAUVET, C. Evidence of limited gene flow in three species of coral reef fishes in the lagoon of New Caledonia. Marine Biology. v. 130, n. 3, p. 361-368 fev.1998.

ROCHA, L. A.; BASS, A. L.; ROBERTSON, D. R.; BOWEN Brian, W. Adult habitat preferences, larval dispersal, \& the comparative phylogeography of three Atlantic surgeonfishes (Teleostei: Acanthuridae). Molecular Ecology. V. 11, p. 243-252, 2002

ROSA, R. S.; MOURA, R. L. Community structure of reef fish in the Biological Reserve of Atol das Rocas, off Brazil. Canada. In : Abstracts A.S.I.H. Annual Meeting, 1995, Edmonton, Canada.

ROSA, R. S.; MOURA, R. L.Visual assessment of reef fish community structure in the Atol das Rocas Biological Reserve, off Northeastern Brazil. In: International Coral Reef Symposium, 8th., 1997. Proceedings International Coral Reef Symposium, v. 1, p. 983-986, 1997

Tropical Oceanography, Recife: v. 31, n. 2, p. 171-180, 2003 
SALE, P. F. The ecology of fishes on coral reefs. Oceaonography Marine Biology. v. 18, p. 367421, 1980.

SMITH, A. J. \& MORAIS, J. O. Estudos preliminares sobre a geologia ambiental costeira do estado do Ceará, nordeste do Brasil. Arquivos de Ciências do Mar, Fortaleza, v.23, p.85-96. 1984.

SMITHIES, O. An improved procedure for starch gel eletrophoresis: furtyer variation in the senem Proteins of normal individuals. The Biochemical Journal, v. 71, p. 585-587, Mar. 1959.

SOLÉ-CAVA, A. M.; THORPE, J. P. Genetic differentiation between morphotypes of the marine sponge Suberites ficus (Demospongiae: Hadromerida). Marine Biology. v. 93, p. 247-253, 1986.

STEVENSON, D. E.; CHAPMAN, R. W.; SEDBERRY, G. R. Stock identification in Nassau grouper, Epinephelus striatus, using microsatellite DNA analysis. Proceedings of the Gulf and Caribbean Fisheries Institute. v. 50, p. 727-749, 1998.

SWOFFRD, D. L. E SELANDER, R. B. BIOSYS-1 programme for the comprehensive analysis of eletrophoretic data in population genetics and sytematics. J. Hered., Washington, v. 72, p.281-283, 1981.

THORPE, A. M.; SOLÉ-CAVA, A. M. The use of allozyme electrophoresis in invertebrate systematics. Zoologica Scripta. v.23, n. 1, p. 3-18, 1994

THORPE, J. P., SOLÉ-CAVA, A. M.; WATTS, P. C. Exploited marine invertebrates: genetics and fisheries. Pp. 165-184 in A. M. Solé-Cava, C. A. M. Russo \& J. P. Thorpe, eds. Marine Genetics. Kluwer Academic Publishers, Amsterdam. 2000.

WAPLES, R. A multispecies approach to the analysis of gene flow in marine shore fishes.

Evolution. v. 41, p. 385-400, 1987.

WARD, R. D. Genetics in fisheries management. Pp. 191-201 in A. M. Solé-Cava, C. A. M. Russo \& J. P. Thorpe, eds. Marine Genetics. Kluwer Academic Publishers, Dordrecht. 2000.

WARD, R. D.; SKIBINSKI, D. O. F. Observed relationships between protein heterozygosity and protein genetic distance and comparisons with neutral expectations. Genet. Res. Camb. V. 45, p. 315-340, 1985.

WHITLOCK, M. C.; McCauley, D. E. Indirect measures of gene flow \& migration: FST is different from 1/(4Nm+1). Heredity. v. 82, p. 117-125, 1999

WRIGHT, S. Evolution and the genetcs of populations. The University of Chicago Press, London, 1978. 